\title{
Effect of Beach-Chair Position on Cerebral Blood Flow in Patients Undergoing Shoulder Surgery-A Preliminary Observational Study
}

\author{
Jesudoss Antony ${ }^{1}$ Georgene Singh ${ }^{2} \quad$ Bijesh Yadav ${ }^{3}$ \\ ${ }^{1}$ Department of Anaesthesia, Christian Medical College, Vellore, \\ India \\ ${ }^{2}$ Department of Neuroanaesthesia, Christian Medical College, \\ Vellore, India \\ ${ }^{3}$ Department of Biostatistics, Christian Medical College, \\ Vellore, India \\ ${ }^{4}$ Anaesthesia and Intensive Care Medicine, Maidstone and \\ Tunbridge Wells NHS Trust, United Kingdom
}

J Neuroanaesthesiol Crit Care 2022;9:16-20.

\begin{abstract}
Keywords

- cerebral blood flow velocity

- beach chair position

- transcranial doppler

Background Hypotension and cerebral hypoperfusion, commonly encountered in beach-chair position under general anesthesia, carry the risk of neurologic complications. There is a paucity of data on monitoring cerebral perfusion. Our objective was to compare the mean arterial pressure (MAP) and middle cerebral artery velocity (Vmca) in the supine and beach-chair position and estimate its correlation during hypotension. Materials and Methods Twenty ASA class I and II patients undergoing elective shoulder surgery in beach-chair position were included in the study. MAP was measured invasively with the pressure transducer leveled to the phlebostatic axis. Vmca was measured with a $2 \mathrm{MHz}$ transcranial Doppler (TCD) probe through the temporal window. Both MAP and Vmca were measured at baseline after anesthetic induction in the supine position (BL), on assuming the beach-chair position (AP), at steady-state hemodynamics in beach-chair position (P1), whenever there was a drop in MAP > 20\% (P2), and on the restoration of MAP (P3).

Results A mean decrease in MAP and Vmca by $24.76 \%$ and $27.96 \%$, respectively, from supine to beach-chair position with a significant linear correlation between MAP and Vmca along with a Pearsons' coefficient of 0.77 was seen. A change in MAP of $1 \mathrm{~mm}$ of $\mathrm{Hg}$ resulted in a change in Vmca by $0.53 \mathrm{~cm} / \mathrm{sec}(p<0.05)$.

Conclusion A significant decrease in MAP and Vmca was observed in the beach-chair position. TCD could be used as a point-of-care noninvasive technique to reliably assess cerebral perfusion.
\end{abstract}

Address for correspondence Sajan Philip George, MD, Department of Anaesthesia, Christian Medical College, Vellore, India (e-mail: drsajanphilip@gmail.com).

\section{Introduction}

Shoulder surgeries are commonly performed in beach-chair positions because of better surgical access to both the

published online October 24, 2021 ISSN 2348-0548 anterior and posterior parts of the shoulder region, lesser arm traction, and a decreased risk of brachial plexus

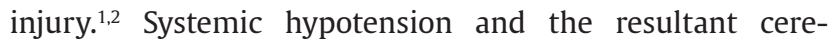
bral hypoperfusion are significant hemodynamic changes
(C) 2021. Indian Society of Neuroanaesthesiology and Critical Care. All rights reserved.

This is an open access article published by Thieme under the terms of the Creative Commons Attribution-NonDerivative-NonCommercial-License, permitting copying and reproduction so long as the original work is given appropriate credit. Contents may not be used for commercial purposes, or adapted, remixed, transformed or built upon. (https://creativecommons.org/licenses/by-nc-nd/4.0/).

Thieme Medical and Scientific Publishers Pvt. Ltd. A-12, 2nd Floor, Sector 2, Noida-201301 UP, India 
observed in the beach-chair position. ${ }^{3-5}$ The effects of an upright posture combined with the effects of general anesthesia exacerbate cerebral hypoperfusion and can potentially lead to cerebral ischemia.

Although the brain can effectively autoregulate its flow and perfusion within a range of mean arterial pressure (MAP) between 50 and $150 \mathrm{~mm} \mathrm{Hg}$, it may not be the same for all individuals. Since the cerebral perfusion pressure (CPP) depends on the MAP, it is imperative to maintain an adequate perfusion pressure at the level of the major cerebral vessels (circle of Willis) to maintain good cerebral perfusion. ${ }^{6}$

One of the noninvasive methods of measuring cerebral blood flow is transcranial Doppler (TCD). The middle cerebral artery (MCA) blood flow velocity (Vmca measured by TCD) can be used as a surrogate to estimate cerebral blood flow (CBF).,8 To our knowledge, there is a paucity of data in the Indian population on the effect of beach-chair position on cerebral perfusion, as assessed by the Vmca. We hypothesized that there will be a decrease in the MAP in the beach-chair position and an associated decrease in the Vmca. The primary objective of our study was to compare the correlation between MAP and Vmca in the supine and beach-chair position in patients undergoing shoulder surgery and to see if the correlation was maintained during episodes of hypotension. The secondary objective was to determine if restoration of the MAP to baseline would restore the CBF, as assessed by the Vmca.

\section{Materials and Methods}

This is a prospective observational study done in a tertiary care center after approval by the institutional review board and ethics committee (IRB Min No:9136 [OBSERVE] dated June 2015).

A total of 20 American Society of Anaesthesiologists (ASA) class 1 and 2 patients, between 18 and 70 years of age, scheduled for elective shoulder surgery in the beach-chair position were included in the study. All eligible patients were included in the study after a detailed explanation of the conduct of the study by the principal investigator, and written informed consent was obtained. The exclusion criteria included patient refusal, ASA III and above, vascular and valvular heart disease, history of cerebrovascular events or traumatic brain injury, patients who are on psychoactive drugs and antiepileptics, sepsis, and pregnancy.

All eligible patients underwent a thorough pre-anesthetic assessment by the primary investigator the day before surgery. Standard fasting guidelines were followed. Reflux prophylaxis with omeprazole $20 \mathrm{mg}$ and anxiolytic premedication with Diazepam $0.1 \mathrm{mg} / \mathrm{kg}$ were administered orally 2 hours before the procedure, as per the standard protocol.

On arrival at the holding bay, the patients were reviewed by the attending anesthesiologist before transfer to the theater. In the operating room, standard ASA monitoring was established with a $\mathrm{SaO}_{2}$, 3 lead ECG, and noninvasive blood pressure (NIBP). An intravenous (IV) access was secured, and the radial artery was cannulated under local anesthesia for the accurate beat-to-beat measurement of MAP on the side opposite to the site of surgery, and the arterial pressure transducer was leveled to the phlebostatic axis in the supine position.

All the patients received balanced anesthesia with standard anesthetic protocol-fentanyl $2 \mathrm{mcg} / \mathrm{kg}$, propofol 2 to $3 \mathrm{mg} / \mathrm{kg}$, vecuronium $0.1 \mathrm{mg} / \mathrm{kg}$ for muscle relaxation, and the trachea was intubated with an appropriate-sized endotracheal tube. Mechanical ventilation was initiated with a tidal volume of 6 to $8 \mathrm{~mL} / \mathrm{kg}$, and anesthesia was maintained with isoflurane (minimum alveolar concentration [MAC] of 0.80.9 ) and $\mathrm{FiO} 2$ of $50 \%$ (air:oxygen). End-tidal carbon-dioxide $\left(\mathrm{EtCO}_{2}\right.$ ) was maintained between 30 to $35 \mathrm{~mm} \mathrm{Hg}$ as per the study protocol. The temperature was monitored using an oropharyngeal temperature probe, and normothermia was maintained with a forced warm air blanket. Blood pressure was maintained within the $20 \%$ range in all patients. Intraoperatively, hypotension was defined as a decrease in the MAP of more than $20 \%$ of the pre-induction value and was treated with IV vasopressors (phenylephrine $50-100 \mathrm{mcg}$ boluses or ephedrine $5 \mathrm{mg}$ boluses) or fluid administration with balanced salt solution/normal saline/Ringer's lactate, as per the discretion of the attending anesthesiologist.

A TCD $2 \mathrm{MHz}$ probe, SonoSite MicroMAXX (SonoSite, Inc. Bothell, WA, USA) was used to measure Vmca through the temporal window, on the side opposite to the shoulder being operated. All recordings were recorded by the principal investigator. The patient was positioned in the beach-chair position, with the head and neck in the neutral position and the torso at 45 degrees. The arterial transducer was positioned at the phlebostatic axis. As per our routine practice, the accompanying hypotension was treated with fluid boluses and vasopressors. No regional anesthesia was given. The patients were extubated in the supine position following the surgery. The patients were followed-up for 48 hours to watch for any neurological complications such as a decrease in sensorium (assessed with Glasgow coma scale [GCS]), focal motor or sensory deficit, and other postoperative anesthetic complications.

\section{Outcome Measures}

All observations were recorded by the attending anesthesiologist from the multiparameter monitor (Intellivue MP 50 Philips Health Care). The TCD was performed by the principal investigator. The variables recorded were MAP, Vmca, heart rate (HR), $\mathrm{SaO}_{2}, \mathrm{EtCO}_{2}$, and MAC. The outcome measures were recorded at baseline after induction of anesthesia and establishing stable ventilation and hemodynamics in the supine position (BL), and soon after assuming the beach-chair position (AP). Once in the beach-chair position, the Vmca was measured at steady-state hemodynamics (P1), whenever there was a significant drop in blood pressure (P2), and thereafter on the restoration of hemodynamics (P3).

\section{Statistical Analysis}

Data was entered using EPI DATA software. Summary statistics were used for reporting demographic and clinical 
characteristics. All categorical variables were reported using frequencies and percentages, and continuous variables were expressed in terms of mean \pm standard deviation (SD) or median (interquartile range [IQR]). For statistical comparisons between the means, a $t$-test was used. The strength of association between MAP and Vmca was tested using the Pearson correlation. Differences were considered significant at $p<0.05$. All the statistical analyses were performed using SPSS 25.0 .

\section{Results}

We screened 26 patients for eligibility and enrolled 22 consecutive eligible, consenting adults scheduled for elective shoulder surgery in the beach-chair position over 1 year. Two subjects were excluded from the analysis since complete data could not be acquired due to technical challenges.

The flowchart of the patients is presented in - Fig. 1. The demographic characteristics of the remaining 20 subjects are presented in $\boldsymbol{- T a b l e ~} \mathbf{1}$. The mean (SD) age of our patients was 37.7 (13.70). Out of a total of 20 patients, the majority were males $(90 \%, n=18)$ and belonged to ASA I $(85 \%, n=17)$. Two patients in ASA II had diabetes mellitus type 2, which was well-controlled on medications.

The percentage change in the MAP and Vmca soon after assuming the beach-chair position from the supine position (supine-AP) is presented in -Table 2. There was a mean decrease in MAP by $24.77 \%$ and a corresponding mean decrease in Vmca by $27.96 \%$. This was within the clinically acceptable range of 20 to $30 \%$. All patients required vasopressors (phenylephrine was used in 18 patients and ephedrine in two patients) and fluid administration to maintain blood pressure during positioning. The beach-chair position resulted in decreases in the MAP and corresponding decreases in Vmca in both groups. There was a negative correlation between age and MAP, which was not statistically significant $(r=-0.26, p=0.28)$. There was no correlation between age and Vmca.

The percentage decrease in MAP and Vmca was compared between the three-time points P1, P2, and P3 which is demonstrated as a box plot in - Fig. 2. Comparison of the mean change in MAP and change in Vmca between these time points (P1 - P2, P2 - P3) is depicted in - Table 3 and does

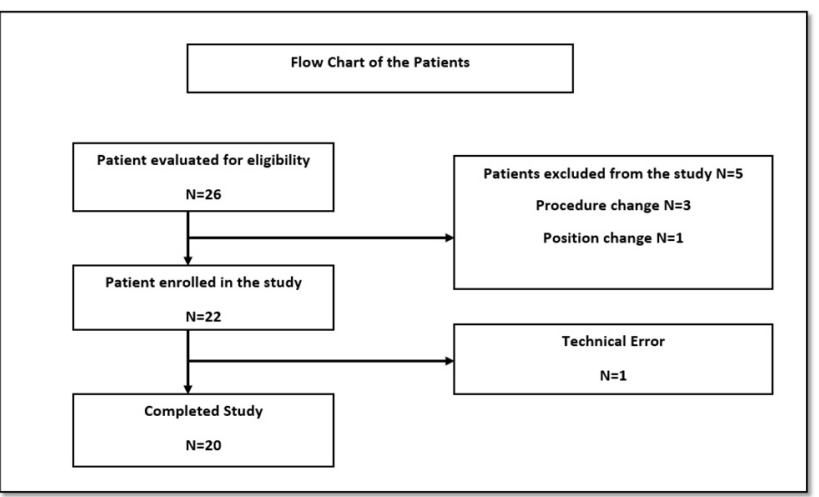

Fig. 1 Flowchart of the patients.
Table 1 Patient characteristics

\begin{tabular}{|c|c|c|}
\hline & Mean & SD \\
\hline Age & 37.60 & 13.71 \\
\hline Weight (kg) & 66.2 & 10.23 \\
\hline Height (cm) & 167.4 & 7.86 \\
\hline BMI $\left(\mathrm{kg} / \mathrm{m}_{2}\right)$ & 23.55 & 2.91 \\
\hline \multicolumn{2}{|c|}{ Patient characteristics } & $n(\%)$ \\
\hline \multirow[t]{2}{*}{ Gender } & Male & $18(90)$ \\
\hline & Female & $2(10)$ \\
\hline \multirow[t]{2}{*}{ ASA } & 1 & 17 (85\%) \\
\hline & 2 & $3(15 \%)$ \\
\hline
\end{tabular}

Abbreviations: ASA, American Society of Anesthesiologists; BMI, bodymass index; SD, standard deviation

Table 2 Decrease in Vmca and MAP on assuming beachchair position

\begin{tabular}{|l|l|}
\hline & Mean \pm SD \\
\hline $\begin{array}{l}\text { Percentage decrease in MAP } \\
\text { (Supine-AP) }\end{array}$ & $24.77 \pm 9.8$ \\
\hline $\begin{array}{l}\text { Percentage decrease in Vmca } \\
\text { (Supine-AP) }\end{array}$ & $27.96 \pm 10.6$ \\
\hline
\end{tabular}

Abbreviations: MAP, mean arterial pressure; Vmca, middle cerebral artery flow velocity; SD, standard deviation.

not show any statistically significant correlation between the two. However, a correlation analysis between the changes in MAP and Vmca at all time points showed a statistically significant linear correlation between the two with a Pearsons' coefficient of 0.77 ( - Fig. $\mathbf{3}$ ). This is demonstrated in $\boldsymbol{\bullet}$ Fig. $\mathbf{3}$. A change in the MAP of $1 \mathrm{~mm}$ of $\mathrm{Hg}$ resulted in a change in the Vmca value by at least $0.53 \mathrm{~cm} / \mathrm{sec}$, which was statistically significant $(p<0.001)$. None of our patients developed any neurological complications during the postoperative period.

\section{Discussion}

The results of our study showed that there was a mean decrease in MAP by $24.77 \%$ and a corresponding decrease in Vmca by $27.96 \%$ in the beach-chair position as compared with the supine position. For procedures on the shoulder, beach-chair position is preferred, as it has been proven to be associated with reduced risk of neurovascular trauma, better visualization, and ease of conversion to an open approach..$^{1-3}$ However, there was a significant decrease in MAP in this position, which was related to the shift of blood induced by the upright posture and the alteration of the cardiovascular reflexes under anesthesia due to the effect of anesthesia. Moreover, the physiological principles involved in the regulation of $\mathrm{CBF}$ such as the cerebral autoregulation and flow metabolism coupling are also altered under anesthesia, contributing to the reduction in $\mathrm{CBF}$, which can potentially give rise to cerebral hypoperfusion and cerebral ischemia in susceptible patients or in those with compromised cerebral circulation. $^{9}$ 


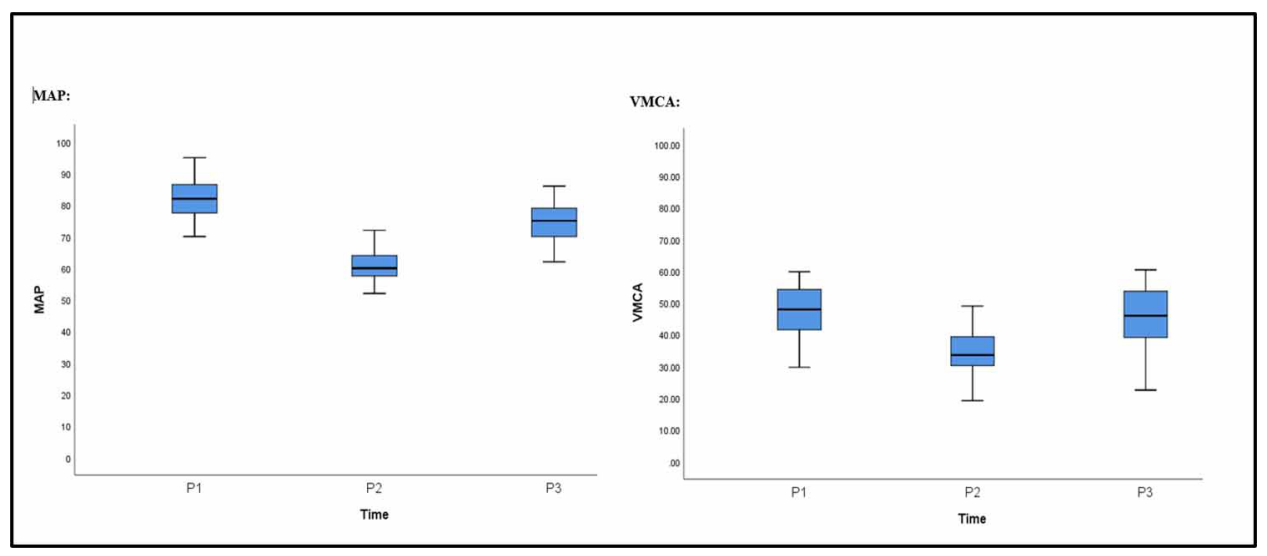

Fig. 2 Box plot showing the comparison of mean arterial pressure (MAP) and middle cerebral artery flow velocity (Vmca) over three time points. $\mathrm{P} 1$-baseline in the beach-chair position at steady state hemodynamics. P2-time of hypotension. P3-time of restoration of blood pressure.

Table 3 Correlation between the change in Vmca with the change in MAP between P1, P2, and P2, P3

\begin{tabular}{|l|l|l|l|}
\hline & Mean \pm SD & \multicolumn{2}{|c|}{ Correlation } \\
\cline { 3 - 3 } & & I & I-Value \\
\hline $\begin{array}{l}\text { Decrease in MAP } \\
(\mathrm{P} 1-\mathrm{P} 2)(n=20)\end{array}$ & $20.7 \pm 8.87$ & 0.14 & 0.55 \\
\hline $\begin{array}{l}\text { Decrease in Vmca } \\
(\mathrm{P} 1-\mathrm{P} 2)(n=20)\end{array}$ & $14.03 \pm 8.02$ & & \\
\hline $\begin{array}{l}\text { Increase in MAP } \\
(\mathrm{P} 2-\mathrm{P} 3)(n=20)\end{array}$ & $15.6 \pm 12.60$ & 0.02 & 0.95 \\
\hline $\begin{array}{l}\text { Increase in Vmca } \\
(\mathrm{P} 2-\mathrm{P} 3)(n=20)\end{array}$ & $11.05 \pm 5.93$ & & \\
\hline $\begin{array}{l}\text { Mean change in MAP } \\
(n=40)\end{array}$ & $2.55 \pm 21.29$ & 0.77 & $<0.001$ \\
\hline $\begin{array}{l}\text { Mean change in Vmca } \\
(n=40)\end{array}$ & $1.48 \pm 14.48$ & & \\
\hline
\end{tabular}

Abbreviations: MAP, mean arterial pressure; Vmca, middle cerebral artery flow velocity; SD, standard deviation.

Note: For one unit change in MAP, the Vmca value significantly increased by 0.53 units $(p<0.001)$.

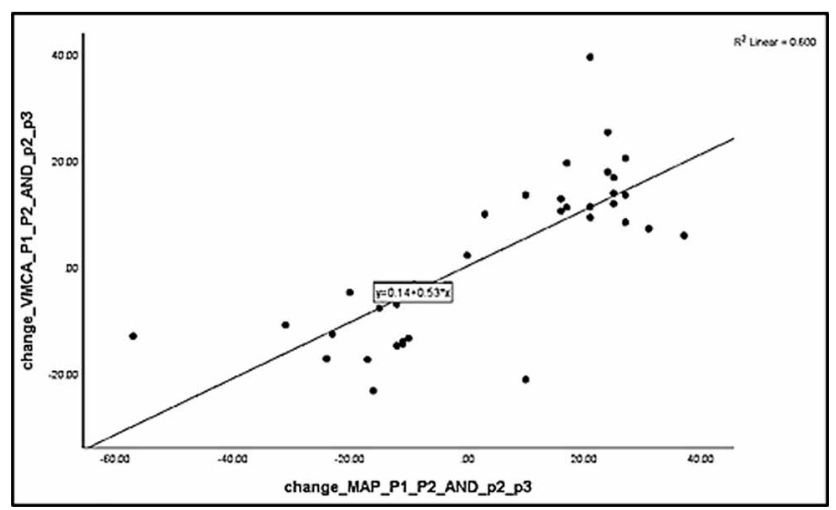

Fig. 3 Correlation analysis between the changes in mean arterial pressure (MAP) and middle cerebral artery flow velocity (Vmca) at all time points.

Although the incidence of perioperative stroke is approximately $1 \%$ in the general surgical population, brain
MRI studies suggest that 1 in 10 elderly patients experience a subclinical perioperative stroke. ${ }^{10}$ There are multiple reports which have shown that neurological deficits do occur due to cerebral ischemia in the sitting and semi-sitting position in patients undergoing general anesthesia. ${ }^{4,5,11}$ This makes it important for us to monitor cerebral perfusion in patients under anesthesia in the beach-chair position.

Monitoring of the cerebral perfusion should be done in all patients at risk of developing cerebral ischemia. TCD measures the flow velocity in the basal cerebral arteries. Although Vmca is not a direct measure of CBF, changes in flow velocity generally correlate with changes in $\mathrm{CBF}$, except in specific situations that can affect MCA diameter such as vasospasm, hypercapnia, migraine, and use of vasoactive agents. This makes it an ideal monitor to measure CBF. ${ }^{9}$ McCullough et al have shown that in anesthetized patients sitting at 45 degrees, there is a mean reduction of $22 \pm 7 \%$ in Vmca as compared with those in the supine position, which is very similar to our results. ${ }^{12}$ Cerebral ischemia has been shown to occur during shoulder surgery in the upright position and this is more so in patients on antihypertensives. ${ }^{4,13}$ Our results have also shown that there is a significant correlation between the changes in Vmca and changes in the MAP, and that Vmca is a sensitive indicator of the changes in $\mathrm{CBF}$ induced by even transient hypotension.

This has also been shown by Newell et al. ${ }^{14}$ The percentage reduction in Vmca that would indicate cerebral ischemia has been studied extensively in carotid endarterectomy, and a reduction in the range of 50 to $60 \%$ has not only been considered as a sensitive indicator for detecting ischemic symptoms but has also been considered as the appropriate threshold for intervention. ${ }^{15}$

Near infrared spectroscopy (NIRS) is being used as a monitor to measure cerebral function, and cerebral desaturations have been reported during shoulder surgeries. ${ }^{16,17}$ However, this is not a commonly available monitor and is not easily available in all centers.

Recently, there has been an increasing availability of point-of-care ultrasound devices, and with the need for rapid 
diagnosis of deteriorating neurologic patients, intensivists are being trained to perform point-of-care TCD at the bedside. ${ }^{18}$ The results of our study show that an ultrasound with a TCD probe can be used as a point-of-care monitor to measure CBF in vulnerable patients intraoperatively and must be used when there is a possibility of cerebral ischemia.

\section{Limitations}

There are several limitations to our study, which include a limited sample size. The posthoc power analysis of the study is only $50 \%$ and is probably due to the limited sample size.

Since we did not have the TCD machine with a specific head frame, we had to insonate intermittently and measure the velocities, which are subjective to change. The baseline velocities were measured only after anesthetizing and not in the awake state, and it was feasible to measure the velocities only on the contralateral side. Other indices such as pulsatility index and resistivity index were not measured. Monitoring the cerebral function using NIRS would have provided objective evidence of cerebral dysfunction, which may correlate with low-flow velocities.

\section{Conclusion}

Our finding suggests that there was a decrease in MAP, which corresponds to decreased Vmca on assuming the beach-chair position. There is also a strong correlation between the changes in blood pressure with the changes in Vmca. An ultrasound with a TCD probe could be used as a point-of-care to assess CBF where conventional TCD is unavailable and must be routinely used in perioperative care, where cerebral blood flow may be compromised as with the sitting or beach-chair position.

\section{Conflict of Interest}

None declared.

\section{References}

1 Skyhar MJ, Altchek DW, Warren RF, Wickiewicz TL, O’Brien SJ. Shoulder arthroscopy with the patient in the beach-chair position. Arthroscopy 1988;4(4):256-259

$2 \mathrm{Li}$ X, Eichinger JK, Hartshorn T, Zhou H, Matzkin EG, Warner JP. A comparison of the lateral decubitus and beach-chair positions for shoulder surgery: advantages and complications. J Am Acad Orthop Surg 2015;23(1):18-28
3 Murphy GS, Greenberg SB, Szokol JW. Safety of beach chair position shoulder surgery: A review of the current literature. Anesth Analg 2019;129(1):101-118

4 Pohl A, Cullen DJ. Cerebral ischemia during shoulder surgery in the upright position: a case series. J Clin Anesth 2005;17(6):463-469

5 Bhatti MT, Enneking FK. Visual loss and ophthalmoplegia after shoulder surgery. Anesth Analg 2003;96(3):899-902

6 Cicolini G, Pizzi C, Palma E, et al. Differences in blood pressure by body position (supine, Fowler's, and sitting) in hypertensive subjects. Am J Hypertens 2011;24(10):1073-1079

7 Christoph NS, Michael EM, Neurologic monitoring. In: Miller RD, ed Miller's Anesthesia 8th ed., Philadelphia, PA: Churchill Livingstone, Elsevier; 2015 1487-1523

8 Ruland S, Aiyagari V. Cerebral autoregulation and blood pressure lowering. Hypertension 2007;49(5):977-978

9 Grüne F, Klimek M. Cerebral blood flow and its autoregulation - when will there be some light in the black box? $\mathrm{Br}$ J Anaesth 2017;119(6):1077-1079

10 Mrkobrada M, Hill MD, Chan MTV, et al. Covert stroke after non-cardiac surgery: a prospective cohort study. $\mathrm{Br}$ J Anaesth 2016;117(2):191-197

11 Morandi X, Riffaud L, Amlashi SFA, Brassier G. Extensive spinal cord infarction after posterior fossa surgery in the sitting position: case report. Neurosurgery 2004;54(6):1512-1515

12 McCulloch TJ, Liyanagama K, Petchell J. Relative hypotension in the beach-chair position: effects on middle cerebral artery blood velocity. Anaesth Intensive Care 2010;38(3):486-491

13 Trentman TL, Fassett SL, Thomas JK, Noble BN, Renfree KJ, Hattrup SJ. More hypotension in patients taking antihypertensives preoperatively during shoulder surgery in the beach chair position. Can J Anaesth 2011;58(11):993-1000

14 Newell DW, Aaslid R, Lam A, Mayberg TS, Winn HR. Comparison of flow and velocity during dynamic autoregulation testing in humans. Stroke 1994;25(4):793-797

15 Halsey JH Jr. Risks and benefits of shunting in carotid endarterectomy. The International Transcranial Doppler Collaborators. Stroke 1992;23(11):1583-1587

16 Moerman AT, De Hert SG, Jacobs TF, De Wilde LF, Wouters PF. Cerebral oxygen desaturation during beach chair position. Eur J Anaesthesiol 2012;29(2):82-87

17 Jeong $\mathrm{H}$, Jeong S, Lim HJ, Lee J, Yoo KY. Cerebral oxygen saturation measured by near-infrared spectroscopy and jugular venous bulb oxygen saturation during arthroscopic shoulder surgery in beach chair position under sevoflurane-nitrous oxide or propofol-remifentanil anesthesia. Anesthesiology 2012;116(5):1047-1056

18 Lau VI, Arntfield RT. Point-of-care transcranial Doppler by intensivists. Crit Ultrasound J 2017;9(1):21 